\title{
Risk of high gestational weight gain on adverse pregnancy outcomes
}

\author{
Rajin Arora $^{1 \#}$, Darin Arora ${ }^{1}$, Jayanton Patumanond ${ }^{2}$ \\ ${ }^{1}$ Department of Obstetrics and Gynecology, Lampang Regional Hospital, Lampang, Thailand \\ ${ }^{2}$ Clinical Epidemiology Unit, Chiang Mai University, Chiang Mai, Thailand \\ Email: "drrajin@live.com
}

Received 11 December 2012; revised 13 January 2013; accepted 22 January 2013

\begin{abstract}
Background/Aims: Excessive gestational weight gain was known to be associated with adverse pregnancy outcomes. It increased the complications during pregnancy, delivery and postpartum period. Nevertheless, there are studies reporting the incompliance of pregnant women with recommendations of weight gain. The objectives of this study were to estimate the prevalence of high gestational weight gain and to identify the increased adverse pregnancy outcomes in these women. Methods: This was a cross sectional study. Data were collected retrospectively from hospital electronic database of Lampang Regional Hospital (LPH) along with manual retrieval from medical charts and labor records. Data of all pregnant women who delivered at labor room of LPH were collected from 1st February 2011 to 31st August 2012. After preterm and multifetal pregnancies were excluded, 4747 cases were brought to the study. This study used the new weight gain recommendation from the Institute of Medicine and National Research Council to classify pregnant women by pre-pregnancy body mass index. Data were analyzed by univariate and multivariate analysis. Results: The proportions of pregnant women with different level of weight gain were $28.4 \%$, 38.5\% and $33.1 \%$ for low, normal and high weight gain. After multivariate analysis was done to control the confounders, women with high weight gain were significantly correlated with having preeclampsia, higher birth weight group, cesarean section and long neonatal length with relative risk (RR) and $95 \%$ confidence interval $(95 \% \mathrm{CI})$ of 4.84 (2.31 - 10.16), 3.94 (3.24 4.79), 2.12 (1.82 - 2.47) and 2.33 (1.90 - 2.86). Conclusions: There were more than half of pregnant women that were prone to have inappropriate weight gain. Many complications from high weight gain that have

*Disclosure: There is no financial or commercial conflict of interest. This paper was granted by Research Committee of Lampang Regional Hospital.

\#Corresponding author.
\end{abstract}

been reported from aboard also occurred in Thai pregnant women. This should alert corresponding health institute to establish a new guideline to avoid high gestational weight gain.

Keywords: Weight Gain; Pregnancy; Prevalence; Pregnancy Outcome

\section{INTRODUCTION}

Gestational weight gain is natural and it is well accepted by pregnant women. In Thailand, low weight gain is obviously concerned by health care providers. But, high weight gain is sometimes seen as appropriate and wealthy by pregnant women and her family. There is a report that high weight gain is common in developed countries [1]. This brings curiosity to investigate its prevalence in developing countries like Thailand. Excessive gestational weight gain along with high pre-pregnancy weight were known to be associated with adverse pregnancy outcomes [2-4]. They increase the risk of complications during pregnancy, delivery and postpartum period [1]. Interestingly, they were usually investigated together in studies. And the conclusions were drawn as co-factors for morbidities. There are only some studies that investigate the effect of gestational weight gain alone.

There were studies stating that, pregnant women with high weight gain had higher risks for preterm delivery, cesarean section, macrosomia, low Apgar score and even offspring overweight/obesity and abdominal obesity in adolescence [5-10], while inadequate weight gain increased asthma, hyperemesis and low birth weight, women with ideal weight gain had less smokers, fetal distress, cesarean delivery and preeclampsia $[10,11]$. Women with excessive rates of gain retained more weight overall, attained a greater postpartum body mass index (BMI) and had higher levels of subcutaneous fat and overweight [11]. Gestational weight gain was also reported as the strongest predictor for sustained weight retention $[6,12]$. There were clinical trials trying to con- 
trol weight gain during pregnancy to avoid such adverse events but definite conclusion is not yet to be known [4].

There had been many recommendations so far, regarding gestational weight gain. The latest and the most popular one was from the Institute of Medicine (IOM) in 2010 [13]. There are studies reporting the compliance of pregnant women with these recommendations of weight gain $[14,15]$. In one study, it reported that mean weight gain in pregnancy was $16.8 \mathrm{~kg}$ [14]. Women gaining weight according to the recommendation of the IOM $(11.5-16.0 \mathrm{~kg})$ had lower frequency of pregnancy and delivery complications than women gaining more than $20.0 \mathrm{~kg}$ [14]. Another study from Canada showed that, $32.6 \%$ of women gained within the recommended guideline, $18.7 \%$ gained below and $48.7 \%$ gained above. The women with lower socio-economic status were more likely to gain above the recommended range. Conversely, being an immigrant, multiparous, and having health problems during pregnancy were associated with less weight gain during pregnancy [15].

In Thailand, there is no study reporting the appropriateness of gestational weight gain of Thai women. And there is no study focused on the sole effect of gestational weight gain on maternal morbidities either. The first objective of this study was to estimate the prevalence of low, normal and high gestational weight gain of pregnant women at Lampang Regional Hospital (LPH). The second objective was to identify the increased proportion of adverse pregnancy outcomes in the high weight gain group. LPH is one of the regional hospitals of Ministry of Public Health in northern Thailand. With the capacity of 800 beds and many excellent centers contained, it very well represents a tertiary care hospital of the country.

\section{MATERIALS AND METHODS}

\subsection{Samples}

This was a cross sectional study. Data were collected retrospectively from hospital electronic database of LPH along with manual retrieval from medical charts and labor records. Inclusion criteria were all pregnant women who delivered at labor room of LPH from 1st February 2011 to 31 st August 2012. There were 5640 cases delivered in that period. Exclusion criteria were cases with incomplete data, preterm and multifetal pregnancy. After cases without pre-pregnancy or pre-delivery weight were excluded, 5583 cases were left. And after preterm and multifetal cases were excluded, 4747 cases were brought to the analysis. Data flow of the study is shown in Figure 1.

\subsection{Data Collection}

Before 2011, maternal pre-pregnancy weight data were not complete. But from 2011, health care personnel of
LPH were encouraged to document pre-pregnancy weight and height data of every pregnant woman at her first antenatal care visit. Data collection of this study were prepregnancy and pre-delivery weight, maternal height along with demographic, obstetric and pregnancy outcomes of both mother and child. This research had been endorsed by Ethics Committee of Lampang Regional Hospital.

\subsection{Definition of Weight Gain}

This study used the new recommendation from the Institute of Medicine and National Research Council for total weight gain during pregnancy classified by pre-pregnancy body mass index [13]. The details of recommendation are in Table 1. Pregnant women then were classified to 3 levels of weight gain. They were low, normal and high weight gain as nominators for the analysis.

\subsection{Analysis Procedure}

In the analysis, the proportions of pregnant women with each level of weight gain were calculated. Demographic, obstetric, complications in pregnancy and pregnancy outcomes of both mother and child were compared among these groups of women. The interested variables were maternal age, maternal height, gravidity, private delivery case, frequency of antenatal care (ANC), health benefit scheme, places of ANC, HIV infection, 1st and 2nd hemoglobin $(\mathrm{Hb})$ test, complication in pregnancy, gesta-

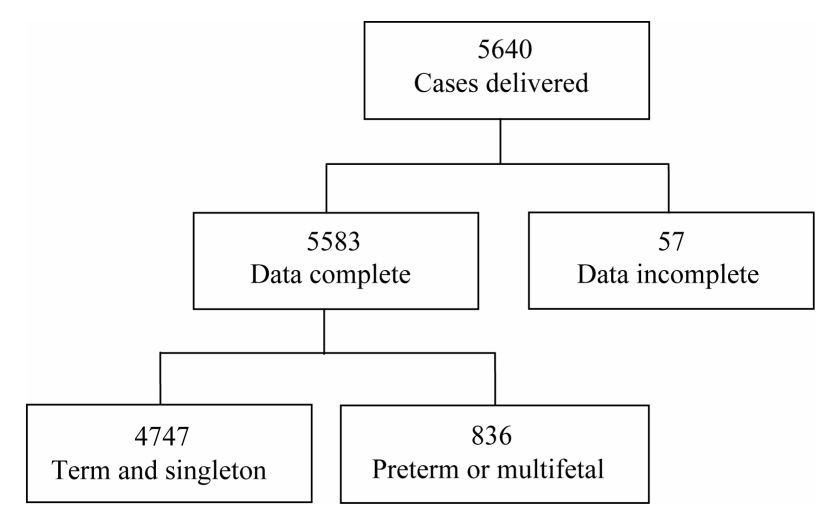

Figure 1. Data flow of the study.

Table 1. Recommendation from the institute of medicine and national research council for total weight gain during pregnancy classified by pre-pregnancy body mass index.

\begin{tabular}{cc}
\hline Pre-pregnancy BMI $\left(\mathrm{kg} / \mathrm{m}^{2}\right)$ & $\begin{array}{c}\text { Recommended total weight } \\
\text { gain range }(\mathrm{kg})\end{array}$ \\
\hline Underweight $(<18.5)$ & $12.5-18.0$ \\
Normal weight $(18.5-24.9)$ & $11.5-16.0$ \\
Overweight $(25.0-29.9)$ & $7.0-11.5$ \\
Obese $(\geq 30.0)$ & $5.0-9.0$ \\
\hline
\end{tabular}


tional age, mode of delivery, presentation, neonatal sex, birth weight, neonatal length and Apgar score.

Data were analyzed in a retrospective cohort approach using standard statistical software. Fisher's exact probability test and student t-test were used where applicable for univariate analysis. Then multivariate analysis was deployed to control the confounders and find the final correlation.

\section{RESULTS}

Within these 19 months period, there were 4747 delivery women with complete data of pregnancy weight gain and interested variables. All cases were delivered at Lampang Regional Hospital. Table 2 shows the proportion of pregnant women with different level of weight gain. There were $28.4 \%, 38.5 \%$ and $33.1 \%$ of women that had low, normal and high weight gain, respectively.

Table 2 also shows the univariate analysis of all demographic and obstetric factors while Table 3 shows complications in pregnancy. They were compared among these 3 groups of women. The analysis showed that high weight gain group had significantly more women with elderly pregnancy. Though mean maternal ages of these 3 groups were comparable, it showed statically significant difference. Proportions of short maternal stature (height $<145 \mathrm{~cm}$ ) were significantly lowest in the high weight gain group. While the proportions of nulliparous women in these 3 groups were comparable.

The proportions of private delivery case and cases with private ANC only were significantly higher in the high weight gain group. In contrary, cases with no ANC and cases with health benefit scheme as universal coverage were significantly lower in high weight gain group. Regarding laboratory tests, high weight gain group significantly had lower proportions of women with HIV infection and anemia from both 1st and 2nd hemoglobin test. In this set of data the results of 1 st routine and 2 nd risk-based VDRL test were all negative.

Regarding complication in pregnancy, low weight gain group had significantly highest proportions of women having 1, 2 and 3 complications. While normal and high weight gain group had comparable proportions of these. The disease-specific analysis showed that high weight gain group had significantly higher proportions of women with preeclampsia but not with antepartum hemorrhage, chorioamnionitis, gestational diabetes, postpartum hemorrhage and premature rupture of membrane.

Table 4 shows the results of univariate analysis of pregnancy outcomes by comparing among the 3 groups of weight gain. These 3 groups had comparable proportions of women with term and postterm birth. Regarding mode of delivery, high weight gain women had significantly more women who delivered by cesarean section. And they also had significantly more women with indication of cephalopelvic disproportion (CPD) and less with the indication of fetal distress. The proportions of vertex presentation and male neonate were comparable among the 3 groups of women.

High weight gain women had significantly higher pro-

Table 2. Demographic and obstetric characteristics classified by levels of weight gain.

\begin{tabular}{lcccc}
\hline \multirow{2}{*}{ Characteristics } & Low & Normal & High & \multirow{2}{*}{ P-value } \\
\cline { 2 - 3 } & $\mathrm{n}(\%)$ & $\mathrm{n}(\%)$ & $\mathrm{n}(\%)$ & \\
\hline Prevalence & $1346(28.4)$ & $1829(38.5)$ & $1572(33.1)$ & \\
Maternal age (year) & & & & $<0.001$ \\
$\quad<20$ & $257(19.1)$ & $233(12.7)$ & $164(10.4)$ & \\
$20-34$ & $905(67.2)$ & $1380(75.5)$ & $1259(80.1)$ & \\
$\geq 35$ & $184(13.7)$ & $216(11.8)$ & $149(9.5)$ & 0.003 \\
$\quad$ Mean \pm SD & $26.3 \pm 6.8$ & $27.1 \pm 6.2$ & $26.9 \pm 5.6$ & $<0.001$ \\
Short stature & $55(4.1)$ & $20(1.1)$ & $10(0.6)$ & 0.081 \\
Nulliparous & $605(45.0)$ & $870(47.6)$ & $763(48.5)$ & $<0.001$ \\
Private delivery case & $425(31.6)$ & $844(46.2)$ & $799(50.8)$ & $<0.001$ \\
No ANC & $34(2.5)$ & $20(1.1)$ & $10(0.6)$ & $<0.001$ \\
Universal coverage & $674(50.1)$ & $762(41.7)$ & $620(39.4)$ & $<0.001$ \\
Private ANC only & $339(25.2)$ & $596(32.6)$ & $589(37.5)$ & 0.007 \\
HIV & $15(1.1)$ & $16(0.9)$ & $4(0.3)$ & $<0.001$ \\
Anemia & & & $186(11.9)$ & $<0.001$ \\
First Hb test & $304(22.9)$ & $304(16.7)$ & $118(11.6)$ & \\
Second Hb test & $179(20.0)$ & $196(16.4)$ & & \\
\hline
\end{tabular}


Table 3. Complications of pregnancy classified by levels of weight gain.

\begin{tabular}{lcccc}
\hline \multirow{2}{*}{ Complications } & Low & Normal & High & P-value \\
\cline { 2 - 4 } & $\mathrm{n}(\%)$ & $\mathrm{n}(\%)$ & $\mathrm{n}(\%)$ & \\
\hline Items & & & & \\
\multicolumn{1}{c}{1} & $601(44.7)$ & $736(40.2)$ & $562(35.8)$ & $<0.001$ \\
2 & $176(13.1)$ & $150(8.2)$ & $134(8.5)$ & $<0.001$ \\
3 & $38(2.8)$ & $25(1.4)$ & $27(1.7)$ & 0.012 \\
Preeclampsia & $20(1.5)$ & $33(1.8)$ & $61(3.9)$ & $<0.001$ \\
Antepartum hemorrhage & $10(0.7)$ & $11(0.6)$ & $7(0.5)$ & 0.586 \\
Chorioamnionitis & $1(0.1)$ & $1(0.1)$ & $4(0.3)$ & 0.296 \\
Gestational diabetes & $58(4.3)$ & $74(4.1)$ & $61(3.9)$ & 0.843 \\
Postpartum hemorrhage & $9(0.7)$ & $21(1.2)$ & $15(1.0)$ & 0.402 \\
Premature rupture of membrane & $35(2.6)$ & $61(3.3)$ & $43(2.7)$ & 0.418 \\
\hline
\end{tabular}

Table 4. Pregnancy outcomes classified by levels of weight gain.

\begin{tabular}{|c|c|c|c|c|}
\hline \multirow{2}{*}{ Pregnancy outcomes } & Low & Normal & High & \multirow{2}{*}{ P-value } \\
\hline & $\mathrm{n}(\%)$ & $\mathrm{n}(\%)$ & $\mathrm{n}(\%)$ & \\
\hline \multicolumn{5}{|l|}{ Gestational age (weeks) } \\
\hline $37-41$ & $1338(99.4)$ & $1815(99.2)$ & $1562(99.4)$ & \multirow[t]{2}{*}{0.823} \\
\hline$\geq 42$ & $8(0.6)$ & $14(0.8)$ & $10(0.6)$ & \\
\hline Cesarean section & $368(27.3)$ & $655(35.8)$ & $754(48.0)$ & $<0.001$ \\
\hline \multicolumn{5}{|l|}{ Indications } \\
\hline Previous cesarean & $102(27.7)$ & $166(25.3)$ & $182(24.1)$ & \multirow[t]{3}{*}{$<0.001$} \\
\hline $\mathrm{CPD}$ & $144(39.1)$ & 314 (47.9) & $418(55.4)$ & \\
\hline Fetal distress & $37(10.1)$ & $40(6.1)$ & $38(5.0)$ & \\
\hline Vertex presentation & $1298(96.5)$ & $1781(97.5)$ & $1522(96.9)$ & 0.118 \\
\hline Male neonate & $687(51.0)$ & $981(53.6)$ & $840(53.4)$ & 0.295 \\
\hline \multicolumn{5}{|l|}{ Birth weight (gram) } \\
\hline$<2500$ & $119(8.9)$ & $86(4.7)$ & $40(2.6)$ & \multirow[t]{3}{*}{$<0.001$} \\
\hline $2501-3499$ & $1123(83.6)$ & $1483(81.2)$ & $1126(71.7)$ & \\
\hline$\geq 3500$ & $102(7.6)$ & $258(14.1)$ & $405(25.8)$ & \\
\hline Mean \pm SD & $2956.0 \pm 366.0$ & $3092.9 \pm 369.1$ & $3239.0 \pm 411.8$ & $<0.001$ \\
\hline Long neonatal length ${ }^{*}$ & $145(10.8)$ & $280(15.4)$ & $364(23.2)$ & $<0.001$ \\
\hline \multicolumn{5}{|l|}{ Placental weight (gram) } \\
\hline Mean \pm SD & $258.2 \pm 91.5$ & $257.6 \pm 99.7$ & $254.1 \pm 114.1$ & 0.497 \\
\hline \multicolumn{5}{|l|}{ Apgar score 0 - 3} \\
\hline 1 minute & $6(0.4)$ & $4(0.2)$ & $3(0.2)$ & 0.347 \\
\hline 5 minutes & $3(0.2)$ & $1(0.1)$ & $1(0.1)$ & 0.644 \\
\hline 10 minutes & $0(0.0)$ & $3(0.2)$ & $1(0.1)$ & 0.341 \\
\hline
\end{tabular}

portions of neonates with birth weight $\geq 3500$ gm. This is also true when comparing mean birth weight among these 3 groups and found that high weight gain group had significantly highest mean neonatal birth weight. With mean of neonatal length of 49.9 and SD of 1.8, neonatal length was categorized as long neonatal length when it was $\geq 52$ $\mathrm{cm}$. High weight gain women had significantly higher proportion of neonates with long neonatal length. These 3 groups of women had comparable mean placental weight and comparable proportions of neonates with se- 
vere birth asphyxia (Apgar score $0-3$ ) at 1,5 and 10 minute.

After univariate analysis was done for every interesting variable, multiple logistic regression analysis was used in multivariate analysis to control confounders. The results are shown in Table 5. Women with high weight gain were still correlated with having preeclampsia with relative risk (RR) and 95\% confidence interval $(95 \% \mathrm{CI})$ of 4.84 (2.31 - 10.16). While higher birth weight group, cesarean section and long neonatal length were all shown to be correlated with high weight gain with RR of 3.94 (3.24 - 4.79), 2.12 (1.82 - 2.47) and $2.33(1.90-2.86)$ respectively.

\section{DISCUSSION}

This study found that $28.4 \%, 38.5 \%$ and $33.1 \%$ of pregnant women had low, normal and high weight gain, respectively. This should bring a surprising alarm to obstetricians in Thailand. They should realize that more than half of the pregnant women they are offering antenatal care would finally have inappropriate weight gain. This finding concurs with a study from Canada, which showed that, only $32.6 \%$ of women gained weight within the recommended guideline, $18.7 \%$ gained below and $48.7 \%$ gained above the guideline [15].

Obstetric health care givers in Thailand should change their usual practices of gestational weight gain monitoring. They should abandon their traditional wholesale approach of checking the final weight gain before delivery. But should instead adopt the IOM tailor-made approach strictly. Every woman entering the antenatal care clinic should be categorized by her BMI for accurate monitoring of the future weight gain. Weight gain should be followed up visit by visit. And the women with inadequate or excessive weight gain should be encouraged to change their nutritional behaviors professionally.

After univariate analysis, there were many variables that showed significant relations with excessive weight gain. But after multiple logistic regression analysis, only 4 pregnancy outcomes were left significant. They were preeclampsia, higher birth weight group, cesarean sec-

Table 5. Outcomes of pregnancy significantly affected by pregnancy weight gain (in multivariable analysis).

\begin{tabular}{|c|c|c|c|}
\hline Outcome of pregnancy & Risk ratio ${ }^{*}$ & $\begin{array}{l}95 \% \text { Confidence } \\
\text { interval }\end{array}$ & P-value \\
\hline Preeclampsia & 4.84 & $2.31-10.16$ & $<0.001$ \\
\hline Higher birth weight group & 3.94 & $3.24-4.79$ & $<0.001$ \\
\hline Cesarean section & 2.12 & $1.82-2.47$ & $<0.001$ \\
\hline Long neonatal length ${ }^{* *}$ & 2.33 & $1.90-2.86$ & $<0.001$ \\
\hline
\end{tabular}

*From exponential risk (risk ratio) regression adjusted for significant predictors; ${ }^{* *} \geq 52 \mathrm{~cm}$. tion and long neonatal length with relative risk (RR) and $95 \%$ confidence interval $(95 \% \mathrm{CI})$ of $4.84(2.31-10.16)$, 3.94 (3.24 - 4.79), $2.12(1.82-2.47)$ and 2.33 (1.90 2.86) respectively. This concurs with many previous studies [5-10]. This study also posed an interesting finding regarding neonatal length. Long neonatal length was shown to be significantly correlated with high weight gain. This should prompt future studies to focus more on body structures of neonates of high weight gain mothers.

Preterm delivery was previously shown to be related with inappropriate weight gain but it was not studied here [7]. Since, this study focused on term and singleton pregnancy. Future studies should include pregnant women with all gestational age if this pregnancy outcome should be scrutinized. Another variable that was significantly related with high gestational weight gain in previous study but not in this one was low Apgar score [6]. This may be due to inadequate sample size of the study to verify such low incidence morbidity. Future studies with large sample size and long-term cohort follow up should be done if long term effects of excessive weight gain should be studied. These include postpartum weight retention and offspring obesity.

\section{CONCLUSION}

This study added important knowledge for Thai obstetric society. There were more than half of pregnant women that were prone to have inappropriate weight gain during pregnancy. Many complications from high weight gain that have been reported from aboard also occur in Thai pregnant women. This should alert Ministry of Public Health to initiate guidelines not only to prevent small for gestational age from low maternal weight gain as before, but also a new guideline to avoid high gestational weight gain. Since, high weight gain is a modifiable risk factor. Health education before pregnancy, awareness of health care providers, good behavioral counseling and weight control program during pregnancy should help to modify this risk $[1,15,16]$.

\section{REFERENCES}

[1] Ronnberg, A.K. and Nilsson, K. (2010) Interventions during pregnancy to reduce excessive gestational weight gain: A systematic review assessing current clinical evidence using the Grading of Recommendations, Assessment, Development and Evaluation (GRADE) system. International Journal of Obstetrics \& Gynaecology, 117, 1327-1334. doi:10.1111/j.1471-0528.2010.02619.x

[2] Bracero, L.A. and Byrne, D.W. (1998) Optimal maternal weight gain during singleton pregnancy. Gynecologic and Obstetric Investigation, 46, 9-16. doi:10.1159/000009988

[3] Carmichael, S., Abrams, B. and Selvin, S. (1997) The pattern of maternal weight gain in women with good pre- 
gnancy outcomes. American Journal of Public Health, 87, 1984-1988. doi:10.2105/AJPH.87.12.1984

[4] Muktabhant, B., Lumbiganon, P., Ngamjarus, C. and Dowswell, T. (2012) Interventions for preventing excessive weight gain during pregnancy. Cochrane Database of Systematic Reviews, 4, CD007145. doi:10.1002/14651858.CD007145.pub2

[5] Park, J.H., Lee, B.E., Park, H.S., Ha, E.H., Lee, S.W. and Kim, Y.J. (2011) Association between pre-pregnancy body mass index and socioeconomic status and impact on pregnancy outcomes in Korea. Journal of Obstetrics and Gynaecology Research, 37, 138-145. doi:10.1111/j.1447-0756.2010.01332.x

[6] Nohr, E.A., Vaeth, M., Baker, J.L., Sørensen, T.I., Olsen, J. and Rasmussen, K.M. (2008) Combined associations of prepregnancy body mass index and gestational weight gain with the outcome of pregnancy. The American Journal of Clinical Nutrition, 87, 1750-1759.

[7] Rudra, C.B., Frederick, I.O. and Williams, M.A. (2008) Pre-pregnancy body mass index and weight gain during pregnancy in relation to preterm delivery subtypes. Acta Obstetricia et Gynecologica Scandinavica, 87, 510-517. doi:10.1080/00016340801996838

[8] Carnero, A.M., Mejía, C.R. and García, P.J. (2012) Rate of gestational weight gain, pre-pregnancy body mass index and preterm birth subtypes: A retrospective cohort study from Peru. International Journal of Obstetrics \& Gynaecology, 119, 924-935. doi:10.1111/j.1471-0528.2012.03345.x

[9] Laitinen, J., Jääskeläinen, A., Hartikainen, A.L., Sovio, U., Vääräsmäki, M., Pouta, A., et al. (2012) Maternal weight gain during the first half of pregnancy and offspring obesity at 16 years: A prospective cohort study. International Journal of Obstetrics \& Gynaecology, 119, 716-723. doi:10.1111/j.1471-0528.2012.03319.x
[10] Ogunyemi, D., Hullett, S., Leeper, J. and Risk, A. (1998) Prepregnancy body mass index, weight gain during pregnancy, and perinatal outcome in a rural black population. Journal of Maternal-Fetal Medicine, 7, 190-193. doi:10.1002/(SICI)1520-6661(199807/08)7:4<190::AIDMFM5>3.0.CO;2-D

[11] Scholl, T.O., Hediger, M.L., Schall, J.I., Ances, I.G. and Smith, W.K. (1995) Gestational weight gain, pregnancy outcome, and postpartum weight retention. Obstetrics \& Gynecology, 86, 423-427. doi:10.1016/0029-7844(95)00190-3

[12] Rössner, S. (1997) Weight gain in pregnancy. Human Reproduction, 12, 110-115.

[13] Rasmussen, K.M., Abrams, B., Bodnar, L.M., Butte, N.F., Catalano, P.M. and Maria Siega-Riz, A. (2010) Recommendations for weight gain during pregnancy in the context of the obesity epidemic. Obstetrics \& Gynecology, 116, 1191-1195. doi:10.1097/AOG.0b013e3181f60da7

[14] Thorsdottir, I., Torfadottir, J.E., Birgisdottir, B.E. and Geirsson, R.T. (2002) Weight gain in women of normal weight before pregnancy: Complications in pregnancy or delivery and birth outcome. Obstetrics \& Gynecology, 99, 799-806. doi:10.1016/S0029-7844(02)01946-4

[15] Kowal, C., Kuk, J. and Tamim, H. (2012) Characteristics of weight gain in pregnancy among Canadian women. Maternal and Child Health Journal, 16, 668-676. doi:10.1007/s10995-011-0771-3

[16] Di Benedetto, A., D’anna, R., Cannata, M.L., Giordano, D., Interdonato, M.L. and Corrado, F. (2012) Effects of prepregnancy body mass index and weight gain during pregnancy on perinatal outcome in glucose-tolerant women. Diabetes \& Metabolism, 38, 63-67. $\underline{\text { doi:10.1016/j.diabet.2011.07.005 }}$ 